\title{
INVESTIGACIÓN DE LAS IDEAS DE LOS ALUMNOS DE ENSENANZA SECUNDARIA SOBRE LA CORRIENTE ELÉCTRICA
}

\author{
RUIZ SÁENZ DE MIERA, A..$^{(1)}$, ROSADO, L..$^{(2)}$ y OLIVA, J. M..$^{(3)}$ \\ (1) I. B. Silverio Lanza, Getafe. \\ (2) UNED. Facultad de Ciencias. Madrid. \\ (3) I. B. Fuerte de Cortadura, Cádiz.
}

\begin{abstract}
SUMMARY
This paper deals with the ideas on electric current of secondary education (BUP and COU) students who have been given traditional tuition. The study was based on open-choice questionnaires, word-choice tests and concept trees. The results obtained show that the students' experiertial and environmental models acquired from their everyday physical and social experience are preserved throughout their secondary education. However, their vocabulary gradually conforms to the «scientific model» of electric current.
\end{abstract}

\section{INTRODUCCIÓN}

El conocimiento de las ideas de los alumnos en Ciencias, de sus modelos representacionales, constituye la base de cualquier desarrollo curricular y, por supuesto, del aprendizaje posterior. Como dice Ausubel: «De todos los factores que influyen en el aprendizaje, el más importante consiste en lo que el alumno ya sabe» (Ausubel 1983). Acceder a las ideas de los al umnos supone un proceso complejo, existiendo distintos procedimientos desde diversas perspectivas y con objetivos diferentes. Por un lado se dispone de técnicas de entrevistas clínicas y cuestionarios escritos destinados a revelar los conocimientos en acción de los estudiantes, mediante la interpretación y/o predicción de fenómenos (Osborne 1980), Osborne y Gilbert 1980, Novick y Nussbaum 1978 y 1981). Por otro, en la bibliografía podemos también encontrar estrategias de elaboración de mapas o diagramas conceptuales, utilizando como técnicas de trabajo la entrevista clínica (Novak y Gowin 1988), técnicas de asociación de palabras (Preece 1976, Johnstone y Mcynihan 1985, Kempa y Nicholls 1983), definiciones de conceptos (Kempa y Hodgson 1976) y construcción de árboles conceptuales (Mathews et al. 1984, Novak y Gowin 1988).

\begin{abstract}
Algunos autores se han ocupado de las ideas de los alumnos sobre la corriente eléctrica. Osborne (1985) establece la existencia de diversos modelos sobre eI modo en que la corriente fluye por un circuito formado por una pila y una bombilla, en alumnos comprendidos entre 10 y 17 años: unipolar, de corrientes encontradas, de atenuación de la corriente a lo largo del circuito y científico. Shipstone $(1984,1985)$ estudia las opiniones de los alumnos de la escuela secundaria sobre la corriente eléctrica en circuitos que constan de varios elementos. En dichos trabajos se pone de manifiesto que un alto porcentaje de alumnos de todos los cursos, consideran que el brillo de una serie de bombillas situadas consecutivamente va disminuyendo paulatinamente a lo largo del circuito, y que una resistencia sólo es capaz de afectar el brillo de una bombilla cuando es intercalada delante de la misma. Este tipo de razonamiento, según el cual la corriente se comporta como si se agotase a medida que avanza por el circuito, ha sido también observado por Closset (1983) y recibe el nombre de «razonamiento secuencial» por considerar al circuito como una sucesión de elementos dispuestos secuencialmente, sin retroacción, en lugar de considerarlo como un
\end{abstract}


sistema global en el que sus elementos se encuentran en mutua interacción (Viennot 1989). Solomon (1985) investiga el punto de vista de los alumnos de primero y tercero de secundaria (11-12, 13-14) sobre la electricidad, empleando un cuestionario con tres preguntas: ¿qué es la electricidad?, ¿a qué se parece la electricidad?, y ¿dónde hay clectricidad? Los resultados ponen de manifiesto que en ambos grupos existe un tronco común de conocimiento social, con una variación muy pequeña en sus interpretaciones, no apreciándose un avance significativo en las concepciones del grupo de mayor edad. En nuestro país son escasas las investigaciones realizadas en este terreno, los pocos trabajos realizados al respecto parecen haber detectado las mismas deficiencias que en alumnos de otros países (Criado y Merino 1987, Varela et al. 1988, Acevedo 1989).

En este trabajo se estudian las ideas de los alumnos de BUP y COU en torno a la corriente eléctrica, mediante cuestionarios de preguntas de respuesta abierta, tests de elección de palabras y árboles conceptuales.

\section{PARTE EXPERIMENTAL}

\section{Características de la muestra}

La investigación se ha llevado a cabo sobre tres grupos mixtos de alumnos de segundo de BUP, tercero de BUP y COU, con edades comprendidas entre 15 y 19 años. Todos pertenecen al Instituto de Bachillerato Silverio Lanza de Getafe (Madrid), en el que reciben una enseñanza tradicional, con exposiciones del profesor, resolución de ejercicios y problemas numéricos, incluyendo una hora de laboratorio quincenal. El centro está situado en una zona eminentemente industrial de la periferia de Madrid. Sociológicamente, los alumnos pertenecen a la clase media y media baja. En un porcentaje elevado sus padres son obreros con poca cualificación, existiendo también pequeños comerciantes y una minoría de cargos intermedios. En la tabla I se recogen algunas características de los grupos que han intervenido en la investigación.

Tabla I

Caracteristicas de los alumnos sobte los que se ha realizado la investigacion.

\begin{tabular}{|c|c|c|c|c|c|}
\hline cintspo & N. Alumnos & Edad medis & $\begin{array}{l}\text { Hivel de } \\
\text { superiores }\end{array}$ & $\begin{array}{l}\text { dlos de } \\
\text { gedilos }\end{array}$ & $\begin{array}{l}9 \text { padces } \\
\text { prifmarios }\end{array}$ \\
\hline 20 BUP & $3 ?$ & 16,5 & $2 . ?$ & 5.5 & 91,9 \\
\hline 30 BUP & 34 & 17.1 & 2,9 & 14,7 & 82,5 \\
\hline $\operatorname{cod}$ & 36 & 18,3 & 1,4 & $13, B$ & 79,1 \\
\hline
\end{tabular}

Todos los alumnos cursaban la asignatura de Física o Física y Química, aunque en el momento de realizar el estudio todavía no habían llegado al tema de electrici. dad. Por consiguiente las ideas que pusieron de manifiesto sobre la corriente eléctrica, se referían a concepciones pre y postinstruccionales adquiridas en etapas anteriores (García Hourcade y Rodríguez de Ávila 1988). Académicamente, los alumnos de segundo de BUP solo habían estudiado aspectos muy genéricos de electricidad 2 ○ 3 años antes, según su procedencia, en la Educación General Básica; los de tercero de BUP habían tratado la corriente eléctrica el curso anterior y los de COU lo habian hecho en los dos cursos anteriores.

\section{Metodología \\ 1. Recopilación de datos}

La recogida de datos entre los alumnos se ha llevado a cabo en tres fases (cuestionario de introducción, test de selección de palabras y construcción de árboles conceptuales), separadas por períodos de 7 a 10 días, según los grupos, y siempre en el tiempo habitual de clase.

\section{a) Cuestionario introductorio}

Constituido por un párrafo inicial, en el que se informaba a los alumnos de que iban a participar en una investigación cuyo objeto era estudiar sus ideas sobre la corriente eléctrica, y un cuestionario de 9 preguntas con defíniciones, explicaciones y valoración de distintos fenómenos relacionados con la corriente eléctrica. El tiempo para responder al cuestionario fue de 30 minutos.

\section{b) Test de selección de palabras}

A partir de los resultados obtenidos en el cuestionario de introducción, la bibliografía al respecto (Osborne y Freyberg 1985, Solomon et al. 1985), y de los conceptos seleccionados por un grupo de profesores del centro, junto a otros extraídos del programa de Física de estos niveles, se elaboró una lista de 103 palabras con el siguiente contenido: conceptos fundamentales de la corriente eléctrica, magnitudes eléctricas, unidades eléctricas, conceptos sacados de otras partes de la electricidad (campoeléctrico, clectromagnetismo), palabras del lenguaje coloquial relacionadas con la electricidad, conceptos y términos de otras partes de la Física y de la Química, y nombres de aparatos de medida de magnitudes físicas. A cada alumno participante en la investigación se le ofreció esta lista ordenada alfabéticamente, pidiéndoles que marcasen las 20 palabras que considerasen fundamentales para explicar la corriente eléctrica y sus propiedades. El número de 20 es sólo orientativo, admitiéndose también como válidos los cuestionarios que se desviasen de esta cantidad en 203 por exceso o por defecto, pero rechazando aquéllos que Io hicieran en un número mayor. De todos los cuestionarios considerados como válidos en un grupo se seleccionaron las palabras que presentaban una frecuencia superior al $50 \%$. Estas palabras, que denominaremos «universo conceptual» del grupo, se utilizaron en etapas posteriores para construir árboles conceptuales.

\section{c) Obtención de árboles conceptuales}

En primer lugar, se adiestró a los alumnos en la construcción de diagramas arbóreos, mostrándoles distintos ejemplos ya realizados, explicándoles el procedimiento de elaboración y pidiéndoles que realizasen un ensayo a partir de una 
lista de conceptos sobre relaciones familiares (padre, madre, hijo, tío, etc.). A continuación se les ofreció la lista de palabras componentes del unniverso conceptual» del grupo, obtenido en la etapa anterior, y se les pidí que, de forma individual, construyesen un árbol conceptual con ellas. Para esta última tarea dispusieron de un tiempo comprendido entre 35 y 45 minutos, según los grupos.

El procedimiento para la construcción de diagramas arbóreos es el empleado por Mathews et al. (1984).

\section{Obtención de la matriz de aproximación}

a) Lectura de los árboles y obtención de la matriz de aproximación

El procedimiento de lectura de los árboles y de construcción de su matriz correspondiente es el seguido por Mathew et al. (1984).

b) Obtención de la semimatriz de aproximación del grupo

Para obtener la semimatriz de aproximación de cada grupo de alumnos, se sumaron las semimatrices individuales correspondientes a cada integrante del mismo. Los valores de los elementos de la semimatriz resultante fueron normalizados dividiéndolos por el de valor más alto. De esta forma, al final, todos los elementos quedaron comprendidos entre 0 y 1 .

\section{Construcción de diagramas bidimensionales de la matriz de aproximación}

El método empleado para la obtención de estos diagramas constituye una versión modificada del empleado por Mathews et al. (1984).

a) A partir de la semimatriz de aproximación de cada grupo de alumnos se obtuvo una semimatriz simétrica completa, calculando su matriz traspuesta y sumando ambas. En la matriz simétrica obtenida todos los elementos de la diagonal principal permanecen en blanco.

b) Los elementos de la diagonal principal se calculaton sumando todos los elementos de cada fila y situando el resuitado en el elemento de la diagonal correspondiente. Simbólicamente

$$
\mathrm{m}_{\mathrm{ij}}=\sum_{\mathrm{j}=\mathrm{i}}^{\mathrm{n}} \mathrm{m}_{\mathrm{ij}}
$$

siendo n el número de conceptos. La matriz resultante tiene tantas filas y columnas como conceptos forman el árbol conceptual. El valor de un elemento $m_{\mathrm{sb}}$ de esta matriz es una medida del inverso de la relación entre los conceptos A y $\mathrm{B}$. El valor del elemento $\mathrm{m}_{\mathrm{ij}}$ de la diagonal principal determina el orden de colocación del concepto I en el diagrama posterior.

c) Para situar los conceptos de la matriz de aproximación en el diagrama se procedió de la siguiente manera: el concepto correspondiente al mínimo valor de la diagonal principal (p. e., concepto A) se colocó en el origen de un sistema de coordenadas cartesianas. El concepto correspondiente al segundo valor más bajo de dicha diagonal (p. e. concepto B) se colocó en el cje $Y$, a una distancia de $A$ igual al valor del elemento $m_{a b}$ de la matriz. El siguiente elemento (p. e., $\mathrm{C}$ ), tercer valor más bajo de la diagonal principal, se situó en un punto del primer cuadrante, de forma que la distancia $\mathrm{CA}$ fuera igual al elemento $m_{\mathrm{c} 2}$ de la matriz y la distancia CB igual al elemento $\mathrm{m}_{\mathrm{cb}}$.

El siguiente concepto, D, ya no pudo ser situado por este procedimiento, pues, en general, no existe ningún punto tal que su distancia a los tres ya situados coincida de forma exacta con los valores correspondientes de la matriz de aproximación. Por ello, el concepto D se situó en un punto tal que hiciera mínimas las diferencias entre las distancias a los conceptos ya situados (DA, DB y DC) $y$ los valores de la matriz correspondientes $\left(m_{d a}, m_{d b}, m_{d d}\right.$ ). Matemáticamente las coordenadas de tal punto pueden calcularse por el método de mínimos cuadrados haciendo mínima la ecuación:

$$
D=\sum_{k=1}^{n-1}\left(d_{n k}-m_{n k}\right)^{2}
$$

que desarrollando la expresión de la distancia, $\mathrm{d}_{n k}$, entre dos puntos toma la forma:

$$
\mathrm{D}=\sum_{\mathrm{k}=1}^{\mathrm{n}-1}\left[\sqrt{\left(\mathrm{x}_{\mathrm{n}}-\mathrm{x}_{\mathrm{k}}\right)^{2}+\left(\mathrm{y}_{\mathrm{n}}-\mathrm{y}_{\mathrm{k}}\right)^{2}-\mathrm{m}_{\mathrm{n} \mathrm{k}}}\right]^{2}
$$

siendo $\mathrm{k}$ los conceptos ya situados expresados mediante su orden de colocación, n eI número de orden del concepto que se desea situar, $x$ e y sus coordienadas $\mathrm{m}_{\mathrm{nk}}$ el elemento de la matriz correspondiente a la fila del concepto $\mathrm{N}$ y a la columna del concepto $\mathrm{K}$.

Los siguientes conceptos se siguen situando por el mismo procedimiento, ajustando su posición por mínimos cuadrados a la posición de los conceptos ya situados.

\section{RESULTADOS Y DISCUSIÓN}

\section{Cuestionario introductorio}

En la tabla II se resumen los resultados del cuestionario introductorio expresados en \%. Únicamente la primera pregunta podía contestarse eligiendo una opción ( $\mathbf{s} /$ no), las demás eran de respuesta libre, pudiendo presentarse el caso de que un alumno escribiese más de una respues. ta, con lo cual el porcentaje total puede superar el $100 \%$.

Respecto al contenido de la tabla, en la primera pregunta resulta interesante comprobar la evolución de la idea de corriente eléctrica «como algo que fluye», que apenas está presente en segundo, aumenta en tercero y alcanza el máximo en COU, casi un $70 \%$. Las respuestas a la segunda pregunta muestran un gran desconocimiento, 
en los alumnos de todos los cursos, de cuáles son las magnitudes que intervienen en la corriente eléctrica; así como una confusión, que se va consolidando de segundo a COU, entre magnitudes y unidades. En la tercera pregunta se pueden destacar los altos porcentajes que aicanzan las dos primeras contestaciones en todos los cursos, respuestas que corresponden a experiencias vivenciales muy arraigadas en los alumnos; también puede detectarse una deficiencia importante en el conocimiento y utilidad de los aparatos de medida de magnitudes eléctricas; de todos los alumnos, tan sólo un 5,5\% de los de COU considera el amperímetro, como algo adecuado para saber si en un sitio hay corriente eléctrica. En el mismo sentido se aprecia una confusión entre el uso del voltímetro y el amperímetro, que se consolida de segundo a COU. En la cuarta pregunta el sentido de las respuestas es coincidente con el de tas anteriores; por un lado, el porcentaje de alumnos que consideran, de forma explícita, la corriente eléctrica como un flujo de electrones, aumenta sistemáticamente de segundo a COU. Las dos

Tabla II

Resallatem at cuestionario de introducción.

\begin{tabular}{|c|c|c|c|}
\hline \multicolumn{4}{|c|}{$\begin{array}{l}\text { "Significa } 10 \text { mismo da palabra "corriente" en corriente } \\
\text { etetctrica y corriente de agua? }\end{array}$} \\
\hline & 20 BUP & 3. BUP * & $\cos \%$ \\
\hline si & 27,7 & 58.8 & 69,3 \\
\hline No & 58.8 & 35,3 & 39,4 \\
\hline No contesta & 13,9 & 5,9 & 11,2 \\
\hline \multicolumn{4}{|c|}{$\begin{array}{l}\text { ¿Qué magnitudes flsicas conoces relacionadas con la co- } \\
\text { rriente elćctricát }\end{array}$} \\
\hline & 2* BUP $\times$ & $3 \times 8 U P x$ & $\cos x$ \\
\hline Diferencia de potencial & 0 & 23,5 & 19,4 \\
\hline Intensidad & 5.5 & 20.6 & 30,5 \\
\hline Resistencin & 13,9 & 20,6 & 25,0 \\
\hline Patencla & 472 & 26,5 & 8,3 \\
\hline Da uni dades en vez magni tudes & 11,1 & 23.5 & 55,5 \\
\hline Otris respuestas & 55,5 & 17,6 & 11,1 \\
\hline \multicolumn{4}{|c|}{ ¿coms puedes saber si en un "sit1o" hay corr. eléctrica? } \\
\hline & $7.8 \mathrm{BUP} \%$ & $3=$ Bup $\times$ & $\cos x$ \\
\hline Metienso los desos da cal anbre & 47,2 & 41,2 & 27,7 \\
\hline A1 enchurar un aparato funciona & 33,3 & 41,2 & 30,5 \\
\hline Con faparatos cletctricos & 2,7 & $B, 8$ & 0 \\
\hline Con un ampersmetro & 0 & 0 & 5.5 \\
\hline otras respuestas & 29,3 & 2,9 & 21,2 \\
\hline Con un voltimetro & 0 & 14,7 & 22,2 \\
\hline \multicolumn{4}{|c|}{ ¿Qué es para ti la corrlente eléctrica? } \\
\hline & $2=$ BUP $x$ & 31 BUP $x$ & $\cos *$ \\
\hline Permite utilizar aparatos & 25,0 & 17,6 & 11,1 \\
\hline Resuelve problemas vida diaria & 19,4 & 20,6 & 19,4 \\
\hline Movimiento o slujo de cargas & 11,1 & 35,3 & 42,7 \\
\hline Energfa & 30,5 & - & 2,8 \\
\hline Otras, respuestas & 21.2 & 24,7 & 5,5 \\
\hline
\end{tabular}

primeras respuestas, con altos porcentajes, nos remiten de nuevo a una idea utilitaria y vivencial de la corriente eléctrica.

\section{Test de elección de palabras}

La tabla III resume los resultados obtenidos en el test de elección de palabras, en ella aparecen aquéllas que fueron elegidas por más de un $50 \%$ de los alumnos en, al menos, uno de los grupos, indicando el porcentaje de elección en cada caso y ordenadas alfabéticamente.

Tabla III

Resumen de los resultadios at test de elección de palabras.

\begin{tabular}{|c|c|c|c|}
\hline & \multicolumn{3}{|c|}{$\begin{array}{l}\% \text { de alumnos que mar- } \\
\text { can la palabra }\end{array}$} \\
\hline & 28 BuP & $3^{2} \mathrm{BUP}$ & $\mathrm{cos}$ \\
\hline Alta tensión & 63,3 & 33,3 & 26,6 \\
\hline Amperio & 43,3 & 69,7 & 72,2 \\
\hline Asociacion paraleld & 20,0 & 54,5 & 47,2 \\
\hline Cargas electricas & 73,3 & 72,7 & 66.7 \\
\hline Campo eléctrico & 70,0 & 66,7 & 72,2 \\
\hline Circuito & 53,3 & 48,5 & 80,5 \\
\hline Conductor & 38,7 & 51,5 & $72, ?$ \\
\hline Corriente alterna & 73,3 & 66.7 & 62,2 \\
\hline Corriente continua & 53,3 & 63,6 & 80,5 \\
\hline Electrones & 63,3 & 42,4 & $47, ?$ \\
\hline Diferen. potencial & 3,3 & 75,7 & 72,2 \\
\hline Generador & 46,7 & 72,7 & 66,6 \\
\hline Interruptor & 60,0 & 30,0 & 16,6 \\
\hline Intensidad & 23,3 & 69,7 & 63,8 \\
\hline Ley de Consomo & 50.0 & 63,7 & 83,3 \\
\hline Ley de Ohm & $83 ; 3$ & 75,7 & 86,1 \\
\hline Ohmio & 60,0 & 87,9 & 58,3 \\
\hline Pila & 50,0 & 36,4 & 25,0 \\
\hline Potencia & 6,7 & 60,6 & 33,3 \\
\hline Resistencia & 46,7 & 90,3 & 94,4 \\
\hline vatio & 83,3 & 90,9 & 80,5 \\
\hline Voltimetro & 53,3 & 39,4 & 33,3 \\
\hline Asociacion serle & 20,0 & 51,5 & 47,2 \\
\hline
\end{tabular}

De nuevo se observa, que aquellas palabras del lenguaje habitual que forman parte de lo que puede llamarse un modelo «vivencial» de corriente eléctrica, consiguen los porcentajes de elección más altos en segundo, descendiendo al pasar a tercero y COU; tal es el caso de alta tensión, interruptor y pila. A la inversa, palabras con una importancia básica en el modelo "científico" de corriente eléctrica, apenas son elegidas en segundo, aumentando los porcentajes al pasar a tercero y COU. Esto sucede con amperio, conductor, diferencia de potencial, intensidad, y en menor medida, asociación en paralelo, circui- 
to, corriente continua, generador y resistencia. Además, en Ia tabla III se pueden detectar algunas confusiones graves compartidas por porcentajes muy altos de alumnos de todos los grupos. Así ocurre con conceptos como vatio $(83,3 / 90,9 / 80,5)$, Iey de Coulomb $(50 / 69,7 / 83,3)$ o campo eléctrico $(70 / 66,6 / 72,2)$, que presentan porcentajes de elección claramente superiores a su importancia en un modelo científico de corriente eléctrica. También resulta interesante la sustitución que tiene lugar, de segundo a $\mathrm{COU}$, del concepto de pila $(46,7 /$ $36,4 / 25)$ por el más adecuado de generador $(46,7 / 72,2 /$ $66,6)$.

Aunque no aparece en la tabla, resulta indicativo del tipo de modelo de corriente eléctrica presente en los alumnos, el concepto de fuerza electromotriz, que se incluía en la lista de palabras y, de todos los alumnos, únicamente fue elegido por un $5,5 \%$ de los de COU.

\section{Árboles conceptuales}

Se han analizado las características estructurales de los árboles conceptuales dibujados por los alumnos en los distintos grupos, según criterios: Iongitud de la cadena más larga, númerode ramificaciones y tipode ramificaciones (tríples, cuádruples, etc.). En general, Ios diagramas de segundo de BUP resultan menos ramificados, con urta estructura marcadamente lineal. En tercero y COU los diagramas presentan mayor riqueza, con cadenas mís cortas y mayor número de ramificaciones.

En la figura $1 \mathrm{se}$ incluyen tres árboles construidos por alumnos de COU. El A, con una disposición lineal, apenas permite descubrir alguna estructura que denole un modelo de corriente eléctrica; el alumno sitúa los conceptos atribuyéndoles un orden de importancia, pero es incapaz de establecer otro tipo đe relación entre ellos. En segundo se obtuvieron II diagramas de este tipo sobre un total de 22. EI diagrama B supone un estado intermedio; en él se establecen algunas relaciones sicnificativas entre grupos de $2 \circ 3$ conceptos: campo eléctrico-cargas eléctricas-ley de Coulomb, diferencia de potencial como magnitud característica de la corriente alterna y continua, resistencia -ley de $\mathrm{Ohm}$. El árbol C presenta una buena estructuración; todo el diagrama se conforma, acertadamente, en torno al concepto de circuito, sin embargo incurren algunos errores de bulto: la diferencia de potencial se considera únicamente una magnitud del campo eléctrico, la ley de Coulomb se relaciona con la intensidad, la ley de Ohm se relaciona sólo con la resistencia; en $\mathrm{COU}$, de 22 árboles considerados, 15 incurrian en ese mismo error, asociando la ley de Ohm únicamente a la resistencia (curiosamente, en todos los casos la ley de Ohm ocupaba el final de una rama, solamente un alumno asocia la ley de Ohm con resistencia, intensidad y diferencia de potencial).

\section{Matrices de aproximación y representaciones bidimen- sionales}

Las figuras 2, 3 y 4 muestran las matrices de proximidad de cada grupo (calculadas a partir de sus árboles con--
Figura 1

Árboles conceptuales construidos por alumnos de COU.

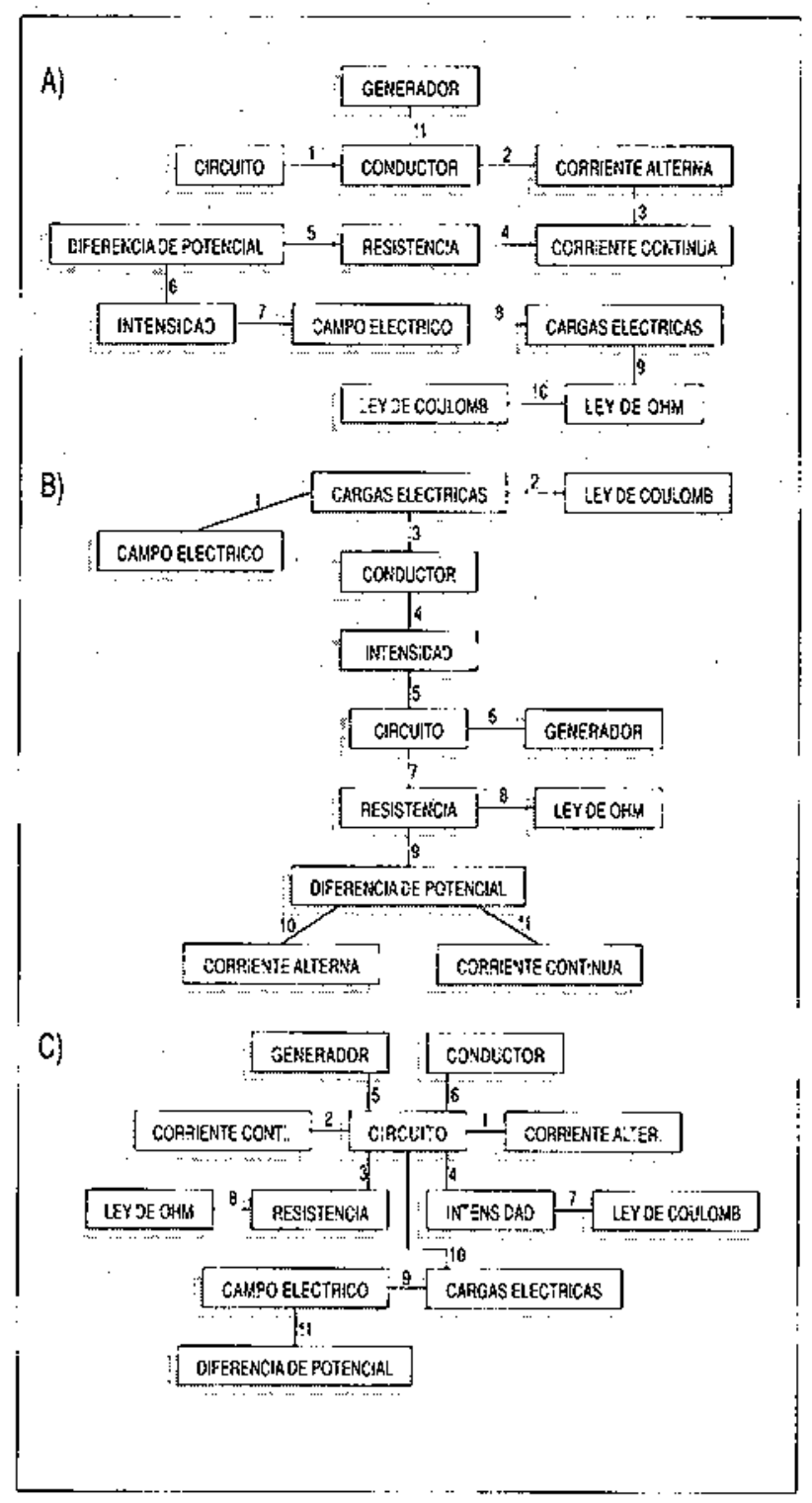

ceptuales) y sus representaciones bidimensionales. Las palabras situadas a la derecha de cada matriz constituyen el «universo semántico» del grupo, formado por las palabras que han elegido más de un $50 \%$ de los alumnos del grupo en el test de elección de palabras, omitiendo aquéllas que fuesen unidades. Se aprecia cómo de segundo a COU el «universo semántico» se va adecuando mejor al utilizado para explicar la corriente eléctrica según un modelo científico; en COU solo sería discutible la presencia de campo eléctrico y ley de Coulomb. Los valores de los elementos de la semimatriz son una medida del inverso de la relación entre los conceptos correspondientes (valores bajos de correlación corresponden a conceptos estrechamente relacionados).

El radio de la circunferencia en los diagramas equivale al valor 1.00 de la matriz. Hasta el concepto 6 o $7 \mathrm{se}$ 
consigue una buena concordancia entre los valores de correlación en la semimatriz y las distancias entre conceptos en el diagrama. A partir del concepto 8 la bondad del ajuste resulta más defíciente. El número situado al lado de cada concepto indica el orden en que ha sido colocado en el diagrama, así como la importancia que atribuyen los alumnos a dicho concepto (números bajos y posiciones centrales indican conceptos relevantes, números altos y posiciones periféricas indican conceptos secundarios. Según esto, para segundo de BUP el concepto más relevante sería cargas eléctricas (fig. 2), mientras que para tercero y COU es circuito (figs. 3 y 4).

Solamente el «universo conceptual» de COU (fig. 4) incluye las magnitudes fundamentales de la corriente eléctrica: resistencia, intensidad y diferencia de potencial; en tercero falta la intensidad (fig. 3) y en segundo no aparece ninguna (fig. 2). Sin ernbargo, la mejora que pueda suponer este hecho en el modelo de corriente eléctrica existente en COU se ve mitigada por la situación poco relevante que ocupan estos conceptos en el diagrama, especialmente intensidad y diferencia de potencial ( 8 y 10 respectivamente).

Para analizar los diagramas de forma más sistemática, se han dividido los conceptos en tres grupos, según su

Figura 2

Matriz de proximidad de segundo de BUP y su representación hidimessional.

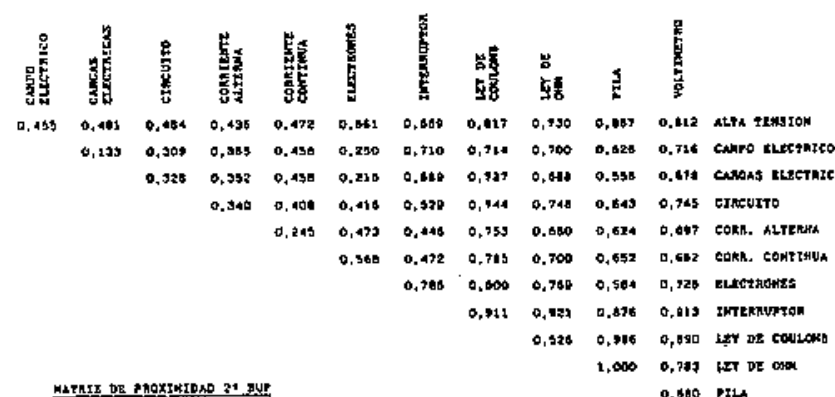

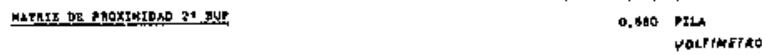

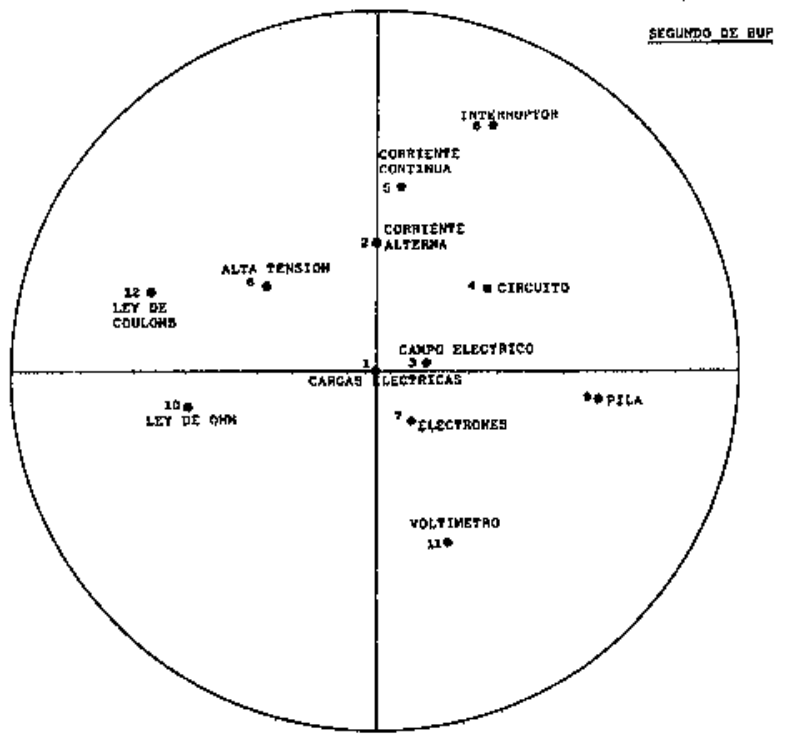

orden de colocación: conceptos importantes (los cuatro primeros), conceptos secundarios (los cuatro últimos) y conceptos intermedios (todos los demás). De acuerdo con tal criterio, no se observan diferencias importantes entre los tres diagramas que permitan descubrir una evolución de los modelos interpretativos de la corriente eléctrica. Sí se advierte, por el contrario, algunas coincidencias que resultan significativas. De los cuatro primeros conceptos situados en los diagramas (conceptos considerados más importantes) tres son comunes a los tres grupos de alumnos (cargas eléctricas, circuito y campo eléctrico). Como ocurría en el test de elección de palabras el campo eléctrico ocupa una posición excesjvamente relevante, situación que se refuerza aún más si tenemos en cuenta que en las tres semimatrices de aproximación, los conceptos más estrechamente relacionados son cargas eléctricas y campo eléctrico, con indices de proximidad de $0,132,0,203$ y 0,282 respectivamente (figs. 2, 3 y 4.) También existe gran sinilitud entre los conceptos situados en los úttimos lugares del diagrama, que en tercero y COU son ocupados por ley de Ohm y ley de Coulomb, mientras que en segundo estos conceptos ocupan los lugares 10 y 12 del diagratma; como ya se puso de manifiesto en la lectura directa de los árboles conceptuales, en ninguno de los grupos de alumnos existe una valoración adecuada del interés de la ley de Ohm para la corriente cléctrica, y erróneamente se equipara en importancia a una ley electrostática como es la ley de Coulomb.

\section{CONCLUSIONES}

Los resultados pueden interpretarse considerando que en los alumnos existen dos modelos de corriente eléctrica, uno «vivencial» o «ambiental», que !es sirve para desenvolverse material y socialmente en aspectos de la vida cotidiana relacionados con la «electricidad», y otro pretendidamente "científico», adquirido durante $\mathrm{el}$ aprendizaje académico. Este último modelo es incipiente en segundo, siendo más rico, con más elementos conceptuales, en tercero y COU; elementos, que por desgracia, presentan bastante indefinición y se encuentran débilmente interrelacionados, conteniendo en muchos casos errores conceptuales graves. El modelo «vivencial» o «ambiental» sigue presente en todos tos cursos.

En los tres cursos el modelo «científico» de corriente eléctrica considera como conceptos fundamentales: cargas eléctricas, circuito y campo eléctrico. Este último denota una deficiencia común a los tres cursos, que establecen una escasa diferenciación entre electrostática y electrocinética, hecho que se manifiesta, también, en la atribución de una importancia análoga para la corriente eléctrica a la ley de Ohm y a la de Coulomb. En segundo todavía no está presente la idea de corriente eléctrica como flujo de cargas, que sí se incorpora de forma generalizada en COU. Ninguno de los cursos considera importante el concepto de fuerza electromotriz, si bien de segundo a COU se incorporan, al menos en su aspecto semántico, resistencia, intensidad y diferencia de potencial. Aunque el desarrollo cognitivo global de los alumnos, a 
Io largo de estos cursos, se traduce en una mayor capacidad en los niveles superiores para organizar la información disponible (construyen árboles de estructura más rica), sólo un alumno en COU ha sido capaz de relacionar correctamente los conceptos de resistencia, intensidad y diferencia de potencial con la ley de Ohm.

En síntesis, no se aprecia una mejoría clara en el modelo de corriente eléctrica de segundo a COU. Sí se detecta un aumento de la precisión semántica. Los alumnos de tercero y COU se encuentran familiarizados con ciertos términos tras haberlos utilizado en cursos anteriores, e incluso son capaces de reconocerlos y de elegir los que más se adecúan a un modelo "científico" de corriente eléctrica, pero no han llegado a comprender su significado preciso y, menos aún, sus interrelaciones; algo típico de un aprendizaje repetitivo de tipo memorístico,

Figura 3

Matriz de proximidad de tercero de BUP y su representación bidimensional.

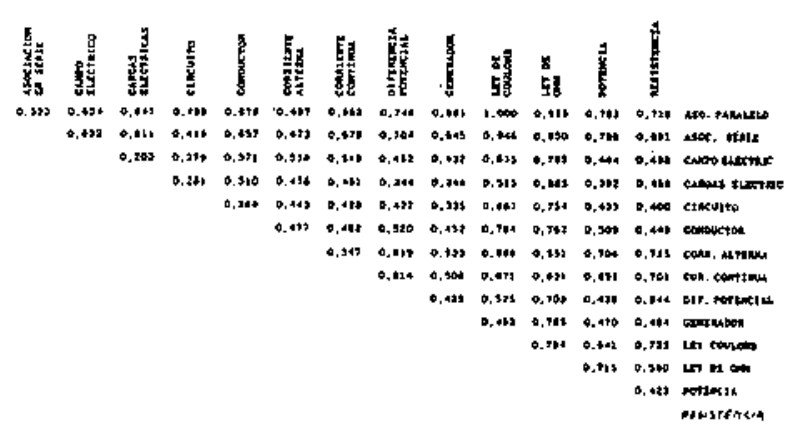

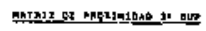

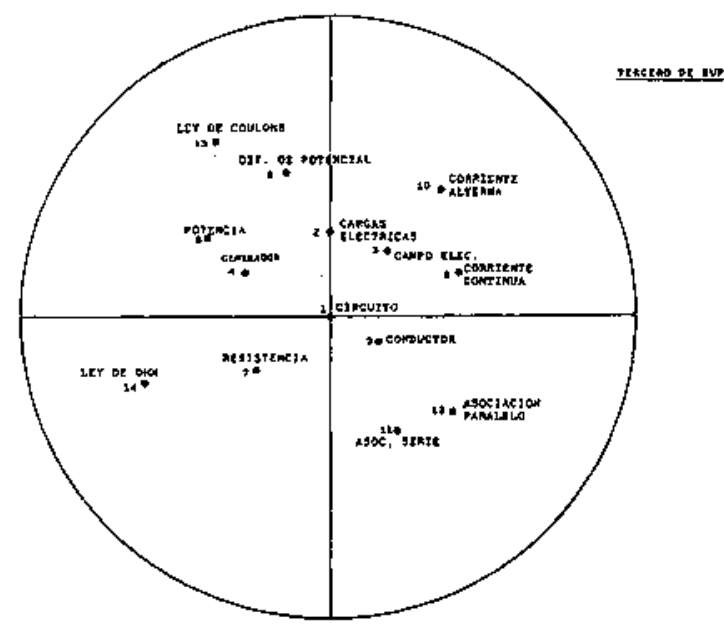

absolutamente alejado del aprendizaje significativo propuesto como paradigma desde todas las instancias que se ocupan de la didáctica de la Física (Driver 19861988 , Novak 1988a, b)

Esperamos que los resultados de esta investigación sir" van como fuente informativa para el profesor que considera el aprendizaje como un proceso que construyen los propios alumnos. Al margen de la dificultad que la corriente eléctrica puede presentar para los estudiantes de estos niveles, consideramos que algunos de los errores conceptuales y deficiencias de aprendizaje puestos de manifiesto, pueden corregirse incluyendo más situa ciones en el aula que impliquen discusion y razonamiento, con un enfoque más globalizador y menos fragmentario del aprendizaje; especialmente en aspectos tan básicos como la diferenciación entre electrostática y electrocinética, o entre magnitudes y unidades.

Figura 4

Matriz de proximicad de COU y su representación bidimensional.

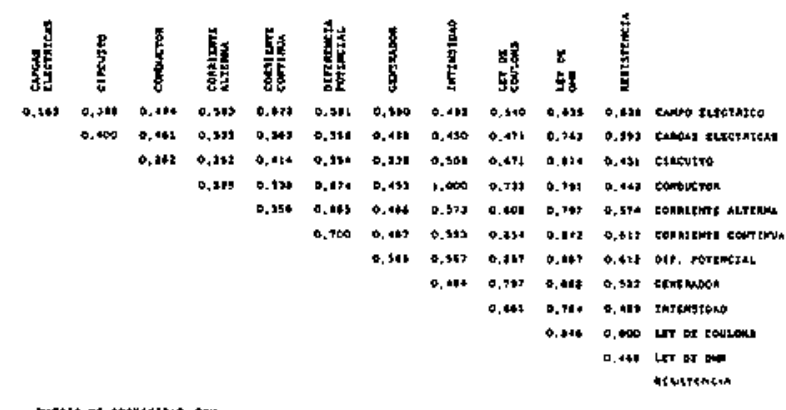

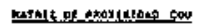

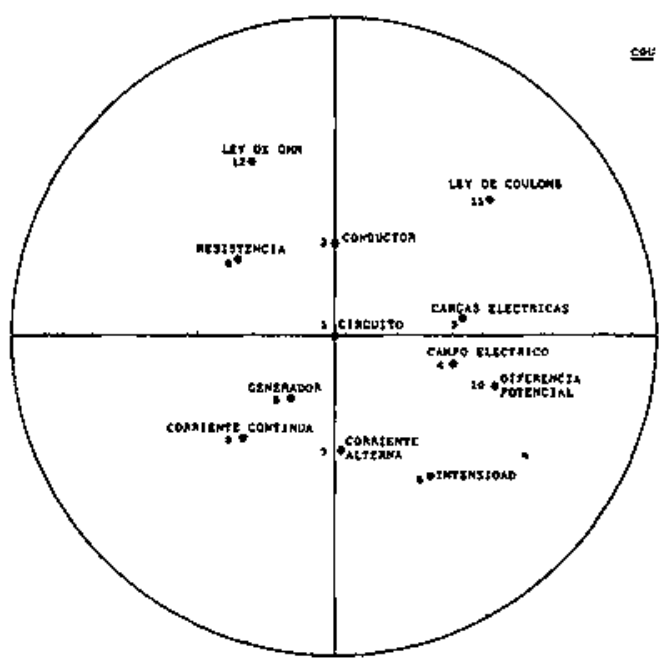




\section{REFERENCIAS BIBL,IOGRÁFICAS}

AUSUBEL, D., NOVAK, J. D. Y HANESIAN, H., 1983. Psicología edicativa. Un punto de vista cognoscitivo. (Trillas: México).

ACFVFDO, J. A., 1989. I as interpretaciones de los estudiantes de BUP sobre electrocinética. Ejemplos con circuitos de corriente continua, Investigación en la escuela, 7 , pp. 107 . 115.

CLOSSET, J., 1983. L e raisonnement secuential en Electrocinetique, Atelier International d'eté: Recherche en Didactique de la Physique. La Londe les Maures.

CRIADO, A. y MERINÓ, J., 1987. Representaciones de los alumnos sobre el comportamiento de circuitos de corriente continua. Actas V Jornadas de Estudio sobre la Investigación en la Escuela. Sevilla. pp. 97-99.

DRIVER, R., 1986. Psicología Cognoscitiva y esquemas conceptuales de los alumnos, Enseñanza de las Ciencias, 4(1), pp. $3-15$.

DRIVER, R., 1988. Un enfoque constructivista para el desarrollo del currículo en Ciencias, Enseñanza de la Ciencia, 6(2), pp. $109-120$.

GARCÍA HOURCADE, J.I. y RODRÍGUFZ DE ÁVILA A, C., 1988. Ideas previas, esquemas alternativos, cambio conceptual y trabajo en el aula, Enseñanza de las Ciencias, 6(2), pp. 161-166.

KEMPA, R. F. y HOIXGSON, G. H., 1976. Levels of concept acquisition and concept maduration in students of chemistry, British Journal of Educational Psychology, 62, pp. 253.

KEMPA, R. F. y NICHOLI.S, C. L., 1983. Problem-solving ability and cognitive structure: an exploratory investigation, European Journal of Science education, 5(2), pp. 171-184.

JOHNSTONE, A.H. y MOYNIHAN, T'.F., 1985. The relationship betwen performances in word association and achievement in chemistry, European Journal of Science Education, 7(1), pp. $57-66$.

MATHEWS, G.P., BROOK, V. G. y KHAN-GANDAPUR, T. H., 1984. Cognitive structure determinations as a tool in science teaching, European Journal of Science Education, $6(2)$, pp. 169

NOVAK, J, D., 1988a. Constructivismo humano: un consenso emergente, Enseñanza de las Ciencias, 6(3), pp. 213-223.
NOVAK, J. D., 1988b. Lerning Science and the Science of leaming, Studies in Science Education, 15, pp. 77-101.

NOVAK, J. D. y GOWIN, D. B., 1988. Aprendiendo a aprender. (Martínez Roca: Barceiona).

NOVICK, S. y NUSSBALM, J., 1978. Junior high school Pupils' understanding of the particulate nature of matter: an interview study, Science Education, 62(3), pp. 273-281.

NOVICK, S. y NUSSBALM, J., 1981. Pupils' understanding of the particulate nature of matter: across-age study, Science education, 65(2), pp: 187-196.

OSBORNF, R. y FREIBERG, P., 1985. I, earning in Science. (Heinemmann: Auckland).

OSBORVL, R. y GLBERT, J., 1980. A method for the investigation of concept understanding in science, European Journal of Science Education, 2(3), pp. 311 .

OSBORNE, R. 1980. Some aspects of students' views of the world, Research in Science Education, 10, pp. 11.

PREECE, P. F. W., 1976. Mapping cognitive stracture: a comparation of method, Journal of Educational Psychology, 68(1), pp. 21 .

RAPOPORT, A., 1967. A comparation of two tree-construction methods for obtaining proximity measures among words, Journal of Verbal Learning Behavior, 6, p. 884.

SHIPSTONE, D., 1984. A study of children's understanding of electricity in simple D.C. circuits, European Journal of Science Education, 6(2), pp. 185-198.

SHIPSTONE, D., 1985. Electricity in simple circuits, en R. Driver (comp.), Children's ideas in science. (Open University Press. Milton Keynes).

SOLOMON, J., 1985. The Pupil's view of clectricity, European Journal of Science Education, 7(3), pp. 281.

VARELA, P., MANRIQUE, M. J. y FAVIGRES, A., 1988. Circuitos eléctricos: una aplicación de un modelo de cnseñanza aprendizaje basado en las ideas previas de los alum. nos, Enseñanza de las Ciencias, 6(3), pp. 285-290.

VIENNO'T, I.,, 1989. La didáctica en la enseñanza superior ¿para qué?, Enseñanza de las Ciencias, 7(1), pp. 3-13.

WATTS, D.M. y ZYYBERSTAIN, J., 1981. A survey of some ideas about force, Physics Education, 16, pp. 360-365. 